\title{
Coordinated lonospheric Reconstruction CubeSat Experiment (CIRCE) mission overview
}

Nicholas, Andrew, Attrill, Gemma D., Dymond, Kenneth, Budzien, Scott, Stephan, Andrew, et al.

Andrew C. Nicholas, Gemma D. R. Attrill, Kenneth F. Dymond, Scott A. Budzien, Andrew W. Stephan, Bruce A. Fritz, Graham J. Routledge, Junayd A. Miah, Charles M. Brown, Peter J. Marquis, Ted T. Finne, Cathryn N. Mitchell, Robert J. Watson, Dhiren O. Kataria, James Williams, "Coordinated Ionospheric Reconstruction CubeSat Experiment (CIRCE) mission overview," Proc. SPIE 11131, CubeSats and SmallSats for Remote Sensing III, 111310E (6 September 2019); doi: 10.1117/12.2528767

SPIE Event: SPIE Optical Engineering + Applications, 2019, San Diego, California, United States 


\title{
Coordinated Ionospheric Reconstruction CubeSat Experiment (CIRCE) Mission Overview
}

Andrew C. Nicholas*a, Gemma D. R. Attrill ${ }^{\mathrm{b}}$, Kenneth F. Dymond ${ }^{\mathrm{a}}$, Scott A. Budzien ${ }^{\mathrm{a}}$, Andrew W. Stephan ${ }^{\mathrm{a}}$, Bruce A. Fritz ${ }^{\mathrm{c}}$, Graham J. Routledge ${ }^{\mathrm{b}}$, Junayd A. Miah ${ }^{\mathrm{b}}$, Charles M. Brown ${ }^{\mathrm{a}}$, Peter J. Marquis $^{\mathrm{a}, \mathrm{d}}$, Ted T. Finne ${ }^{\mathrm{a}}$, Cathryn N. Mitchell ${ }^{\mathrm{e}}$, Robert J. Watson ${ }^{\mathrm{e}}$, Dhiren O. Kataria ${ }^{\mathrm{f}}$, and James Williams $^{\mathrm{g}}$

${ }^{a}$ U.S. Naval Research Laboratory, 4555 Overlook Ave, Washington, DC, 20375, USA; ${ }^{b}$ Defence Science \& Technology Laboratory, Porton Down, Salisbury, Wiltshire, SP4 0JQ, U.K.; 'National Research Council Postdoctoral Research Associate resident at the U.S. Naval Research Laboratory, Washington, D.C., USA; ' now at NASA Wallops Space Flight Facility, Wallops Island, VA, USA;

${ }^{\mathrm{e}}$ Department of Electronic and Electrical Engineering, University of Bath, U.K.; ${ }^{\mathrm{f}}$ Mullard Space

Science Laboratory, University College London, Surrey RH5 6NT, U.K.; ${ }^{\text {g}}$ Surrey Satellite Technologies Limited, Guilford, U.K.

\begin{abstract}
The Coordinated Ionospheric Reconstruction Cubesat Experiment (CIRCE) is a joint US/UK mission consisting of two $6 \mathrm{U}$ CubeSats actively maintaining a lead-follow configuration in the same low Earth orbit with a launch planned for the 2020 timeframe. These nanosatellites will each feature multiple space weather payloads. From the US, the Naval Research Laboratory will provide two 1U Triple Tiny Ionospheric Photometers (Tri-TIPs) on each satellite, observing the ultraviolet $135.6 \mathrm{~nm}$ emission of atomic oxygen at nighttime. The primary objective is to characterize the twodimensional distribution of electrons in the Equatorial Ionization Anomaly (EIA). The methodology used to reconstruct the nighttime ionosphere employs continuous UV photometry from four distinct viewing angles in combination with an additional data source, such as in situ plasma density measurements, with advanced image space reconstruction algorithm tomography techniques. From the UK, the Defence Science and Technology Laboratory (Dstl) is providing the In-situ \& Remote Ionospheric Sensing suite consisting of an Ion/Neutral Mass Spectrometer, a triple-frequency GPS receiver for ionospheric sensing, and a radiation environment monitor. We present our mission concept, simulations illustrating the imaging capability of the Tri-TIP sensor suite, and a range of science questions addressable via these measurements.

Keywords: Ionosphere, Measurement Techniques, Remote Sensing, Tomography, In-situ, Ultraviolet, CubeSat
\end{abstract}

\section{INTRODUCTION}

The U. S. Naval Research Laboratory (NRL) is jointly developing the Coordinated Ionospheric Reconstruction Cubesat Experiment (CIRCE) mission with the UK Defence Science and Technology Laboratory (Dstl). CIRCE is an ionospheric tomography mission targeting multipoint/multi-sensor measurements via a two-satellite constellation consisting of $6 \mathrm{U}$ CubeSats that will fly in a lead-trail configuration. The launch, expected no earlier than January 2020, is supported by the DoD Space Test Program and will insert the spacecraft into circular orbits with an altitude of $500 \mathrm{~km} \pm 10 \mathrm{~km}$ and an inclination of $90^{\circ} \pm 5^{\circ}$. Each of the CIRCE CubeSats will carry a set of remote sensing and in-situ sensors to measure the space environment. The NRL will provide two UV optical remote sensors called Triple Tiny Ionospheric Photometer

*Andrew.nicholas@nrl.navy.mil; phone 1202 767-2441; www.nrl.navy.mil 
(Tri-TIP) for each spacecraft and Dstl will provide the In-situ and Remote Ionospheric Sensing (IRIS) suite, comprising an ion/neutral mass spectrometer (INMS), a GPS radio occultation sensor (TOPCAT) and a radiation monitor (RadMon) for each spacecraft. This paper will provide a CIRCE mission overview with a primary focus on the optical instrumentation.

\subsection{CIRCE Mission Objectives}

The Earth's ionosphere is formed by solar radiation that ionizes the neutral species of the upper atmosphere. Although orders of magnitude less dense than the neutral atmosphere, the ionosphere exhibits significant control of the upper atmosphere through interaction with the ambient electric and magnetic fields. The ionosphere is also responsible for effects on radio waves that bounce, scatter, or are mutated as they pass through this region. Although the global climatology of this plasma layer is relatively well known, its daily evolution exhibits extraordinary variability both spatially and temporally in response to space weather events (e.g. geomagnetic storms, coronal mass ejections, solar flares), to physical and chemical processes, and to dynamics from the lower atmosphere (waves, tides, circulation, meteorological events). Resultant ionospheric structures, particularly vertical and horizontal gradients, can profoundly affect or even disrupt the propagation of electromagnetic radiation. The neutral and ion gases in the Earth's upper atmosphere and ionosphere have airglow signatures visible throughout the day and night that provide the means to remotely sense and specify the ionosphere globally. Many single-satellite missions have been employed in the past to observe these emissions, and thus these regions, to inform our understanding of the structure of the ionosphere. However, these results are restricted in accuracy, cadence, and spatial resolution by the need to make the assumption that the ionosphere is static over the timeframe needed to pass through a region. Coordinated multi-sensor, multi-angle observations provide a data set that minimizes these limitations and thus can be used to reconstruct a given region of the ionosphere with higher accuracy.

The objectives of the CIRCE mission are to accurately characterize the dynamic ionosphere by providing tomographic specification of electron density versus altitude derived from simultaneous ultraviolet (UV) observations of the ionosphere from multiple CubeSats and different view angles, and provide in-situ ionospheric particle and radiation measurements combined with remote-sensing of GPS signals to map the ionosphere.

\section{$1.2 \quad$ Tri-TIP Background}

The lower atmosphere on Earth completely absorbs light at far ultraviolet (FUV) wavelengths. Space-based remote sensing of the upper atmosphere is therefore well suited for the FUV spectral regime due to the lack of background emission from the Earth's surface and lower atmosphere. The F-region of the ionosphere, from $\sim 150$ to $500 \mathrm{~km}$ in altitude, emits light at several discrete FUV wavelengths. In the shadow of the Earth, these emissions are known as nightglow and are often used to characterize the physical state of the ionosphere. ${ }^{1}$ NRL has developed a class of compact, high-sensitivity, remote sensing instruments to measure FUV nightglow and monitor the density and structure of the ionosphere. ${ }^{1,2}$

The Tiny Ionospheric Photometer (TIP) was first developed by NRL to monitor FUV emissions of atomic oxygen in the ionosphere as part of the Constellation Observing System for Meteorology, Ionosphere, and Climate (COSMIC) mission, also known as the Formosa Satellite Mission \#3 (FORMOSAT-3). ${ }^{2,3}$ The F-region ionosphere is composed predominantly of electrons and $\mathrm{O}^{+}$ions $(\sim 99 \%)$ with minor populations of $\mathrm{O}_{2}^{+}, \mathrm{N}_{2}^{+}, \mathrm{N}^{+}, \mathrm{H}^{+}, \mathrm{He}^{+}$, and $\mathrm{NO}^{+}$ions. Nightglow is primarily produced by the decay of ionospheric plasma, especially via radiative recombination (between ions and electrons) and mutual neutralization (between positive and negative ions). In particular, the recombination of $\mathrm{O}^{+}$ with electrons produces neutral oxygen atoms in an excited electronic state. Excited oxygen atoms release photons as they rapidly return to the ground state and emit two of the brightest features in Earth's FUV nightglow spectrum. One feature, a triplet emission at 130.2, 130.4, and $130.6 \mathrm{~nm}$ (hereafter referred to as OI $130.4 \mathrm{~nm}$ ), is difficult to analyze because high levels of atmospheric absorption make it optically thick. The other feature, a spin-forbidden oxygen doublet at 135.6 and $135.8 \mathrm{~nm}$ (referred to as OI $135.6 \mathrm{~nm}$ ), is optically thin and was used by TIP to determine the plasma density of the $\mathrm{F}$ region along the spacecraft nadir. ${ }^{2}$

\subsection{Spacecraft Layout and Viewing Geometry}

The CIRCE CubeSats are 6U spacecraft provided by Blue Canyon Technologies. The spacecraft will be flown in a 3axis stable configuration with the $2 \mathrm{U} \times 1 \mathrm{U}$ section facing into the velocity direction and the $3 \mathrm{U} \times 1 \mathrm{U}$ section facing the nadir direction. The CIRCE CubeSats will be flown in coplanar circular orbits in a lead-trail configuration. The spacecraft bus components are located in the two center units, the IRIS suite is located at the front (ram) of the leading 
spacecraft and the Tri-TIP sensors in the rear (wake) side of the spacecraft and the reverse on the trailing spacecraft, as depicted in Figure 1. The viewing angles of each of the Tri-TIP provide multiple lines of sight through the ionosphere. The lead spacecraft will carry two Tri-TIP sensors, one that looks $45^{\circ}$ down in the wake direction and one that is pointed $17^{\circ}$ down in the wake direction to observe the Earth's limb. The trail spacecraft also carries two Tri-TIP sensors, one that is pointed $45^{\circ}$ down in the ram direction, to sample the same patch of ionosphere as the $45^{\circ}$ down instrument on the lead spacecraft, and one that points in the nadir direction. The in-track separation between the two CIRCE spacecraft, nominally $250 \mathrm{~km}$ to $500 \mathrm{~km}$, has been chosen to optimize the viewing geometry of the four Tri-TIP sensors to provide multi-point sampling of the ionosphere. This baseline separation will be obtained and maintained during the mission via differential drag techniques, i.e. intentionally changing the attitude of the spacecraft to increase or decrease the projected frontal area and thereby alter the force of atmospheric drag on that spacecraft compared to the other CIRCE spacecraft. The Tri-TIP observations are made on the nightside portion of the orbit, and hence to maximize science collection for the INMS and TOPCAT payloads the trail spacecraft will perform a $180^{\circ}$ yaw flip maneuver every other orbit to align the in-situ INMS payload into the ram direction during the sunlit (dayside) portion of the orbit.

\section{Leading Satellite}

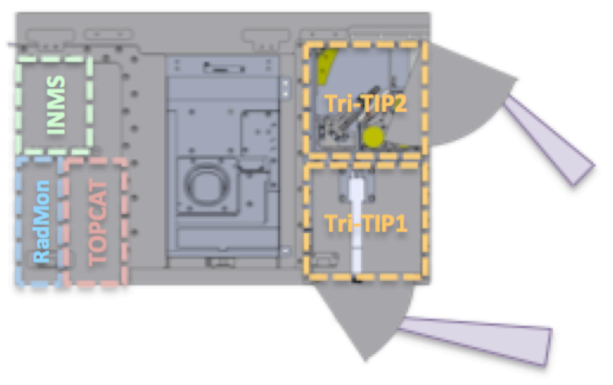

\section{Trailing Satellite}

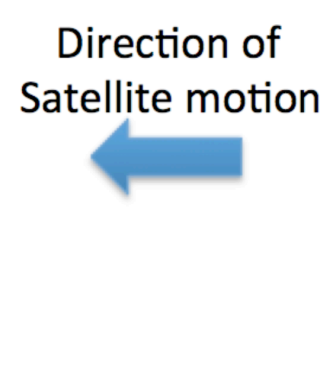

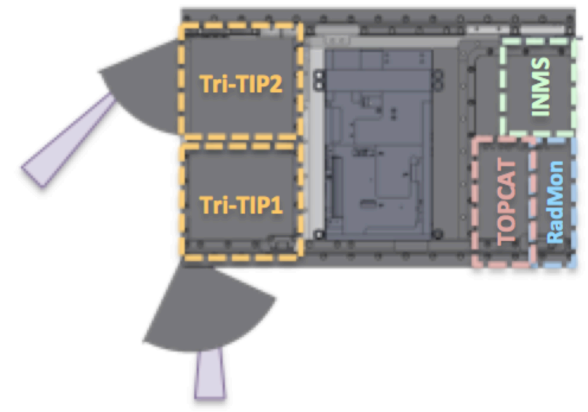

Figure 1. Instrument layout and viewing geometry of the CIRCE spacecraft. The IRIS suite of sensors, INMS, RadMon and TOPCAT, are located in the front 2 Us of the spacecraft the bus avionics and control electronics are in the center 2Us, and the two Tri-TIP sensors are located in the rear 2Us. The trail spacecraft is depicted in the yaw-flipped configuration for nightside tomographic fixed angle observations of the ionosphere by Tri-TIP.

\section{TRI-TIP SENSOR}

\subsection{Layout and optical path}

The Tri-TIP instrument is a CubeSat compatible instrument designed to fit within a $10 \mathrm{~cm} \times 10 \mathrm{~cm} \times 10 \mathrm{~cm}$ (1U) volume. Figure 2 depicts a cross-section of the instrument illustrating the light path through the sensor and also an isometric view with dimensions. The hinged deployable mirror assembly in its stowed configuration acts as a dust cover to keep dust and debris from entering the instrument during storage, testing, shipping and launch. Once deployed, light from the Earth's atmosphere is reflected off the magnesium fluoride $\left(\mathrm{MgF}_{2}\right)$ coated aluminum mirror and enters the instrument through a small two-vane baffle and onto an off-axis parabolic mirror (also with an Al substrate with a $\mathrm{Al} / \mathrm{MgF}_{2}$ coating). The light passes a shutter and solenoid assembly designed to protect the photomultiplier tubes (PMTs) from being exposed to direct sunlight. The shutter solenoid is actuated by a sun-sensor in the electronics stack that is oriented towards the deployed mirror. After passing the open shutter, light passes through a strontium fluoride $\left(\mathrm{SrF}_{2}\right)$ long-pass filter. The remaining light is divided by a beam splitter between two PMTs at the prime focus, directing the half of the light towards the UV PMT and the other half towards the red leak PMT. The three Tri-TIP detectors are Hamamatsu R13194 PMTs driven at $1100 \mathrm{~V}$, to monitor the UV signal, the red leak signal and the dark background (including South Atlantic Anomaly and other high energy radiation). The field of view at the PMT is $3^{\circ} \times 7.25^{\circ}$ (for the $45^{\circ}$ and nadir versions, $0.2^{\circ} \times 7.25^{\circ}$ for the limb version). 

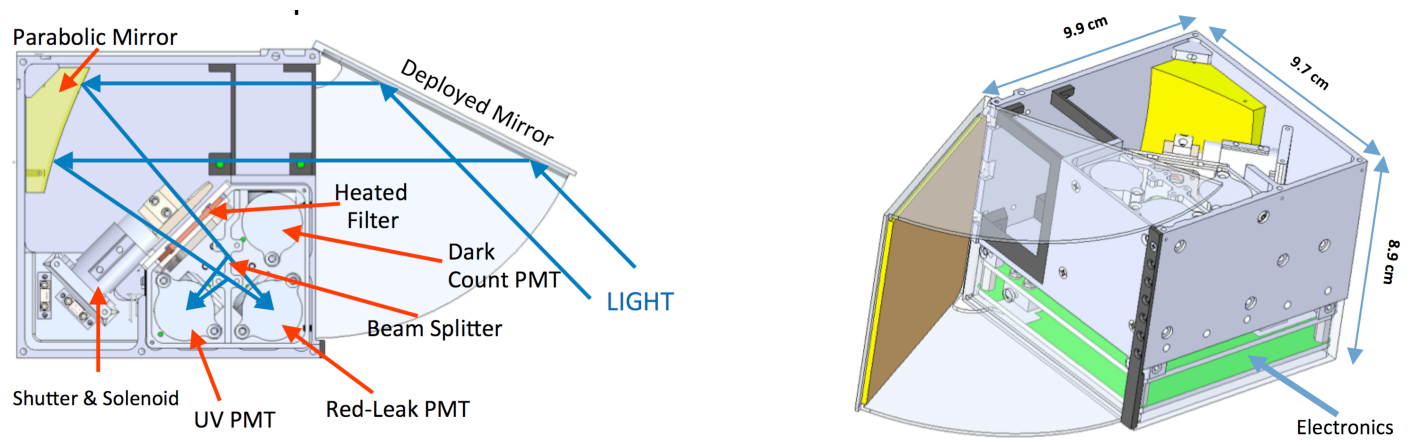

Figure 2. (Left) Top-down view of optical layout of the Tri-TIP sensor. (Right) Isometric view of the Tri-TIP sensor.

\subsection{Heated $\mathrm{SrF}_{2}$ filter}

The SrF2 filter, when heated to $>90 \mathrm{C}$, shifts the filters short wavelength cutoff to a value between $130.4 \mathrm{~nm}$ and 135.6 $\mathrm{nm}$ effectively isolating the desired $135.6 \mathrm{~nm}$ emission from the relatively brighter $130.4 \mathrm{~nm}$ and hydrogen Lyman$\alpha(121.6 \mathrm{~nm})$ emissions. The filter is mounted in an assembly that provides thermal isolation from the aluminum TriTIP chassis and is driven by redundant strip heaters to maintain the required cutoff temperature. The filter housing is shown in Figure 3. Laboratory data was acquired to characterize the filter transmissivity between $128.0 \mathrm{~nm}$ and 137.0 $\mathrm{nm}$ over a range of temperatures to monitor the shift of the short wavelength cutoff of the filter. These data ${ }^{4}$ presented in Figure 4 were used to determine the operating set point $(100 \mathrm{C} \pm 1 \mathrm{C})$ of the heaters. Figure 5 shows the measured transmissivity of the $\mathrm{SrF}_{2}$ filters at both $130.4 \mathrm{~nm}$ and $135.6 \mathrm{~nm}$ versus temperature.

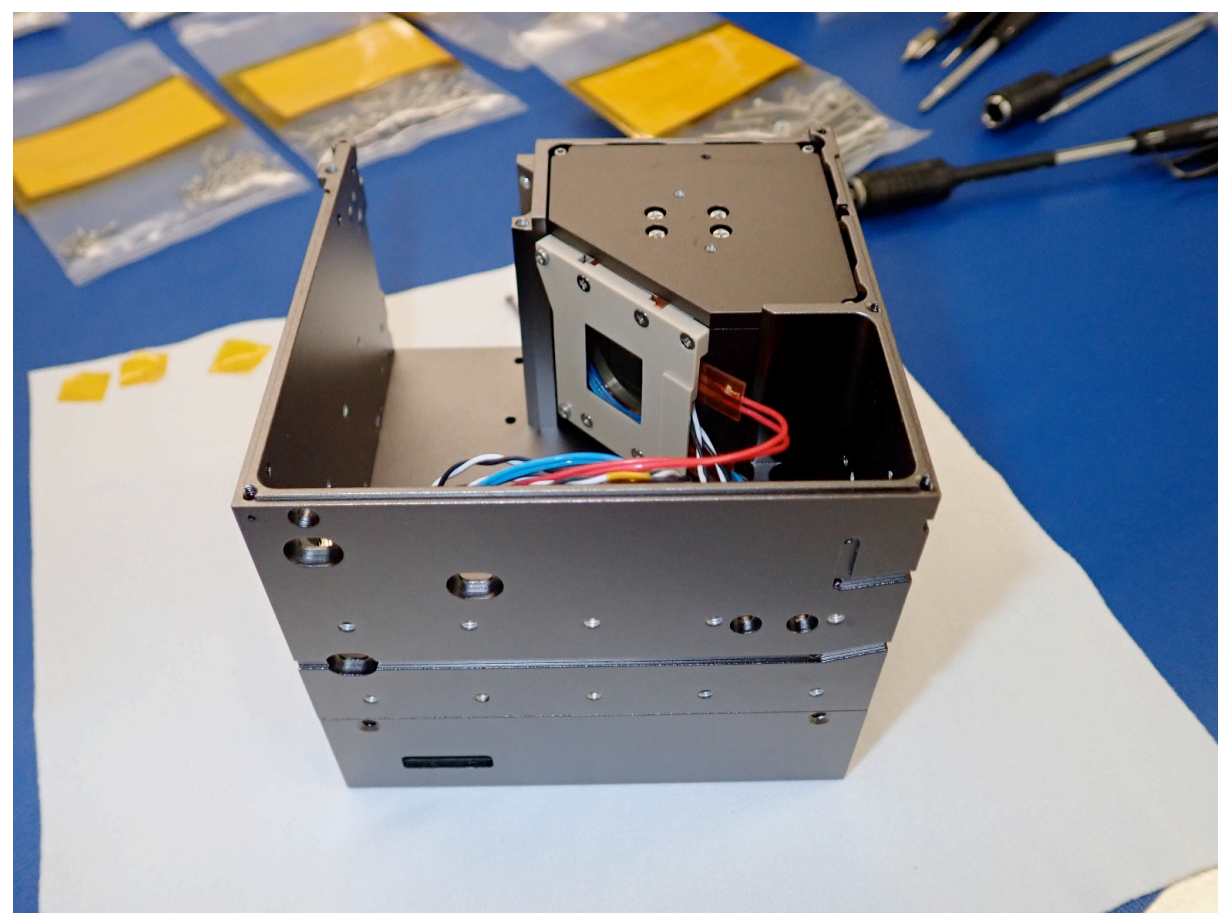

Figure 3. Photograph of the Tri-TIP Chassis with the lid and deployable mirror removed. The heated Strontium Fluoride filter is housed in the beige structure at the center of the photo, the wiring entering the filter assembly provides power to heat the unit and connections to the two thermistors that monitor the temperature of the unit that drive the heater power cycling. 


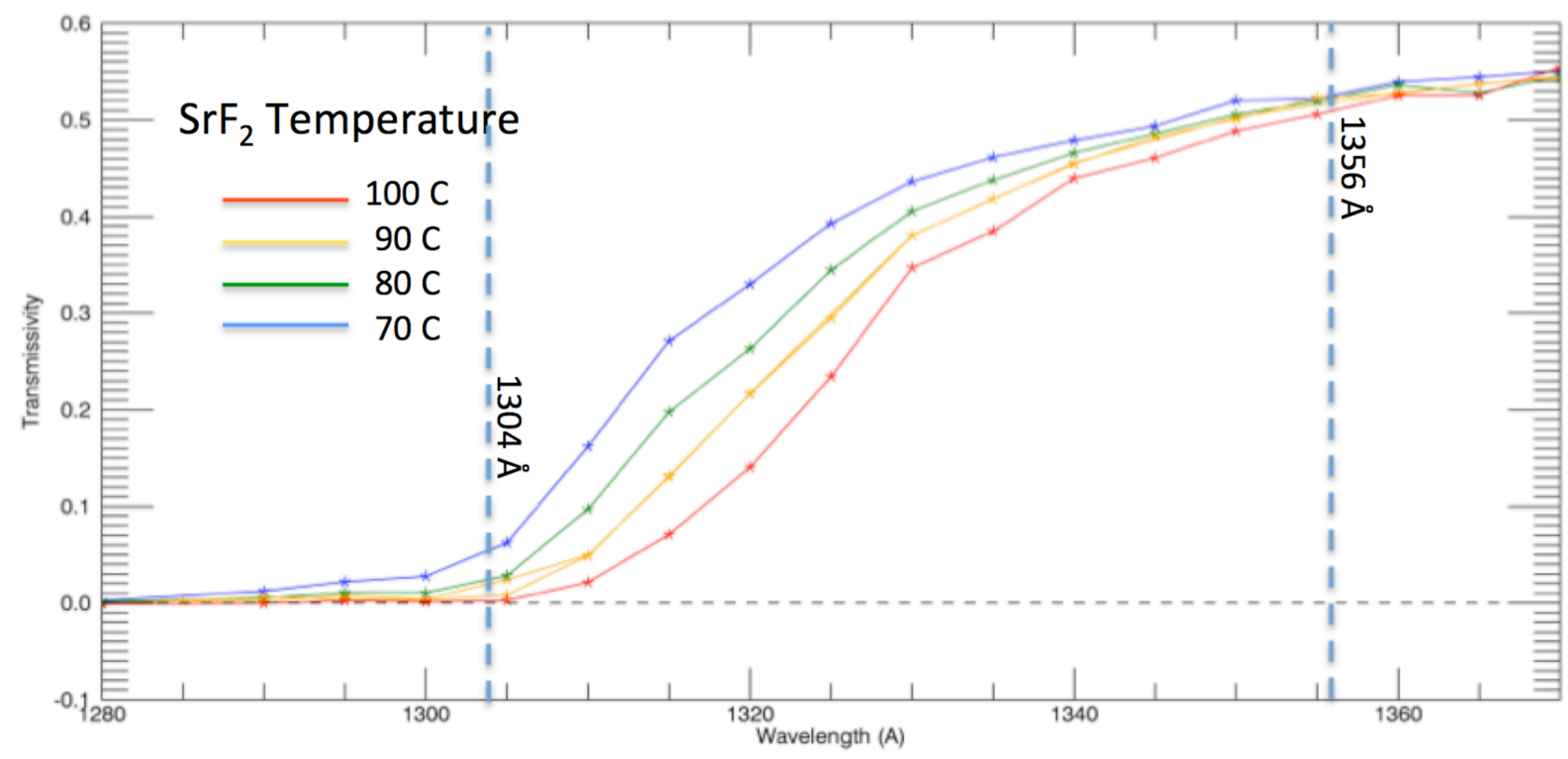

Figure 4. Laboratory data reporting the measured transmissivity of the $\mathrm{SrF}_{2}$ filter with temperature. The wavelengths of interest (130.4 $\mathrm{nm}$ to be suppressed and $135.6 \mathrm{~nm}$ to be passed) are highlighted by dashed vertical lines. ${ }^{4}$

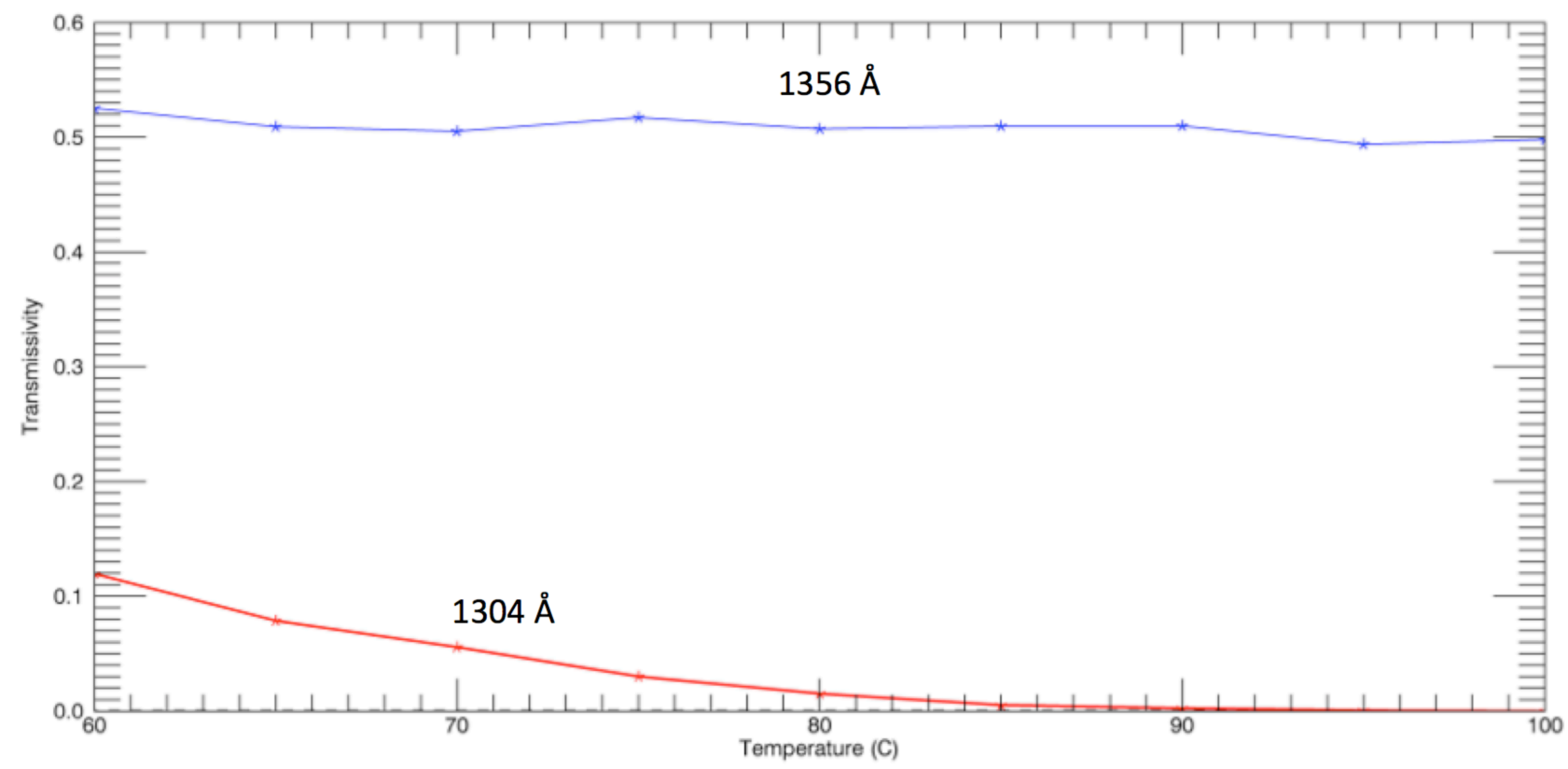

Figure 5. Laboratory data characterizing the transmissivity of the $\mathrm{SrF}_{2}$ filter at $130.4 \mathrm{~nm}$ and $135.6 \mathrm{~nm}$ as a function of temperature. ${ }^{4}$ 


\subsection{Beam splitter}

The Tri-TIP sensor utilizes three separate photomultiplier tubes (PMTs) to measure the UV signal, which can be contaminated by long wavelength scattered light (red leak) from moonlit clouds and/or city lights from the ground, the red-leak signal, and the background dark counts (tube background plus high energy signal from SAA and other sources). A beam splitter is used to direct light to the UV and the red leak tubes while the dark count tube is completely enclosed and not illuminated by light coming through the optical path.

The beam splitter, a Hemlux vacuum ultraviolet grade sapphire $\left(\mathrm{Al}_{2} \mathrm{O}_{3}\right)$ filter, is $2 \mathrm{~mm}$ thick with an outer diameter of 20 $\mathrm{mm}$. The sapphire will transmit the red leak photons but block the UV photons allowing one to effectively measure the red leak contamination observed by the PMTs. A reflective $\mathrm{Al} / \mathrm{MgF}_{2}$ coating is deposited on one surface in a repeating polka dot pattern to achieve a $50 \%$ reflected/throughput ratio. The pattern is repeating circles with a diameter of 1.197 $\mathrm{mm}$ and a center-to-center spacing of $1.5 \mathrm{~mm}$, and sample beam splitter is shown in Figure 6. The laboratory tested throughput and reflectance was measured and plotted $^{5}$ versus the simulated values as presented in Figure 7 . For the limb sensor the tangent point observation will occur near $200 \mathrm{~km}$ altitude, hence there is no expected red leak source from scattered moonlight on clouds or city lights, a $\mathrm{MgF}_{2}$ beam splitter substrate is implemented, this allows the UV portion of the light to reach both PMTs. Due to the light passing through two index of refraction medium changes, one from vacuum to $\mathrm{MgF}_{2}$ as the light enters the beam splitter and one from $\mathrm{MgF}_{2}$ beam splitter back to vacuum as the light exits the beam splitter, one can effectively sample a second altitude with the "red leak" tube by slightly moving the position of its entrance slit in front of the PMT. This allows the CIRCE limb sensor to sample two fields, at $17^{\circ}$ down and $18^{\circ}$ down, which for a $500 \mathrm{~km}$ circular orbit translate to tangent point altitudes of $200 \mathrm{~km}$ and $163 \mathrm{~km}$ respectively. A ray trace of light bundles from these two offset angles are shown in Figure 8.

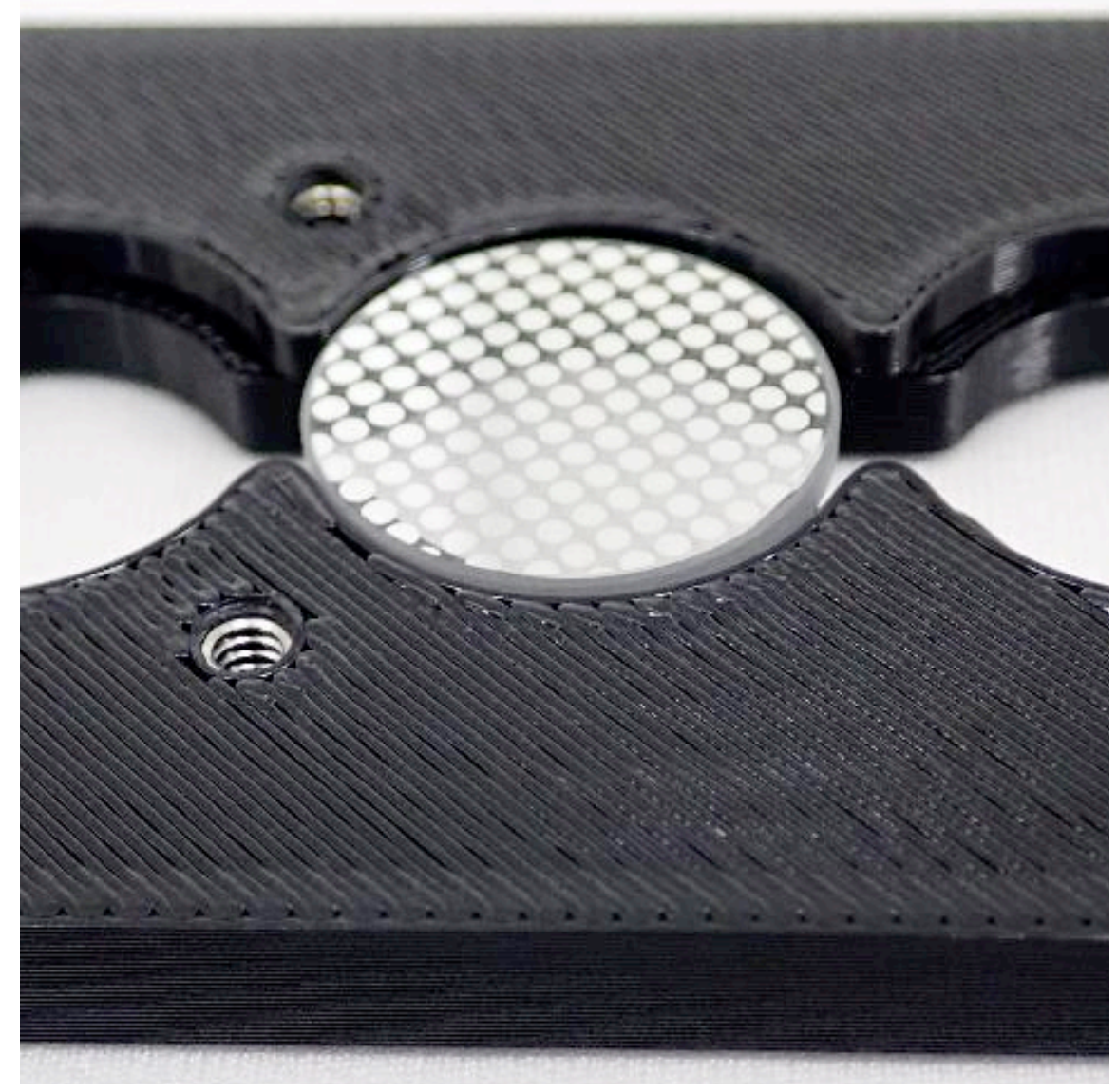

Figure 6. A photograph of one of the beam splitters in the test laboratory frame. 


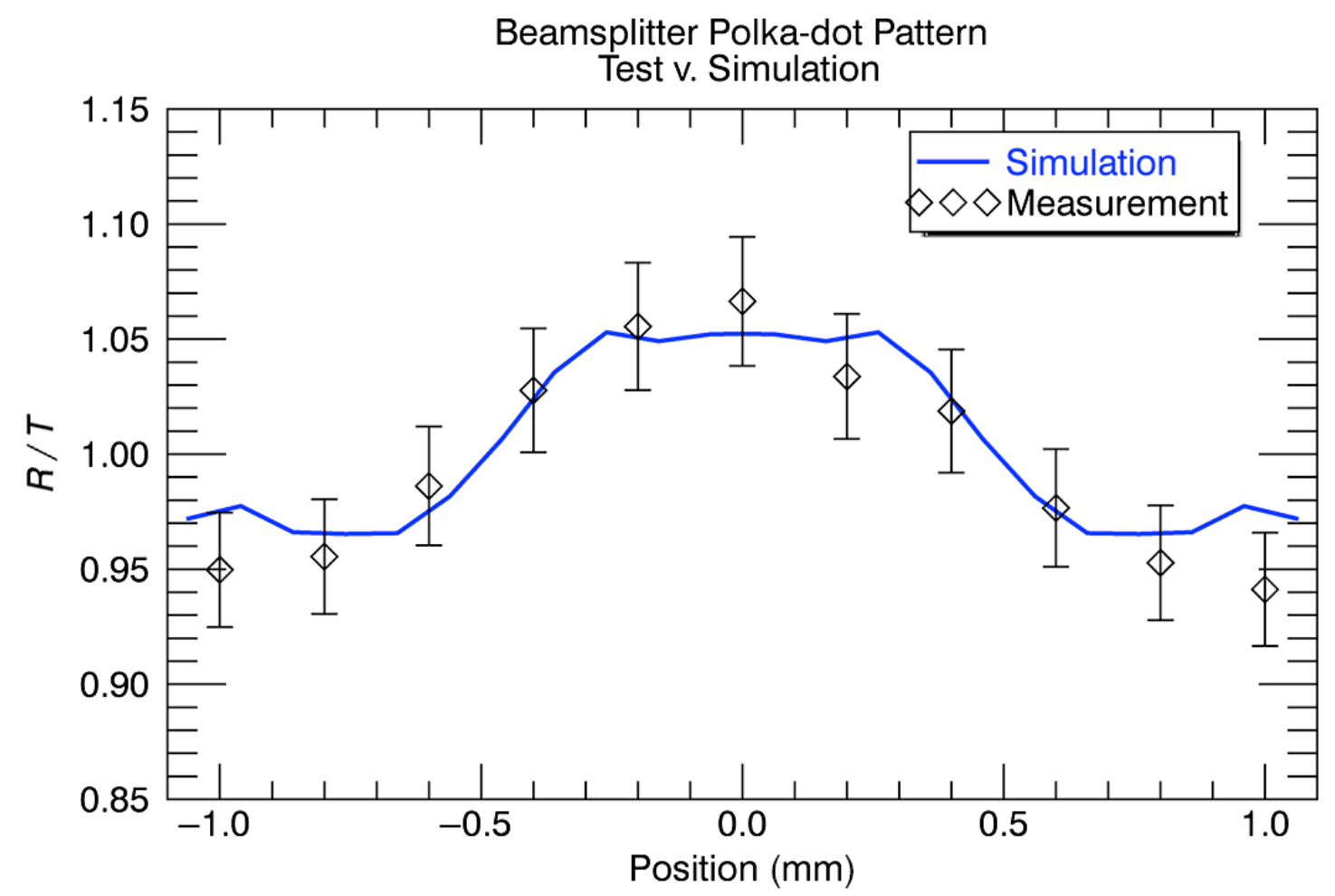

Figure 7. The ratio of measured reflectance to transmissivity of the sapphire beam splitter is represented by the black diamonds. The blue line is a simulation of the geometric effect from scanning the polka dot pattern in $0.1 \mathrm{~mm}$ increments across the center of one of the dots. The $\sim 7 \%$ modulation seen in this test was due to the $5 \mathrm{~mm}$ dia. sample beam. This beam did not cover an integral number of periods of the polka dot pattern, hence the modulation. In flight, the beam splitter will see a $\sim 10 \mathrm{~mm} \times 10 \mathrm{~mm}$ illumination, greatly reducing any modulation. ${ }^{5}$

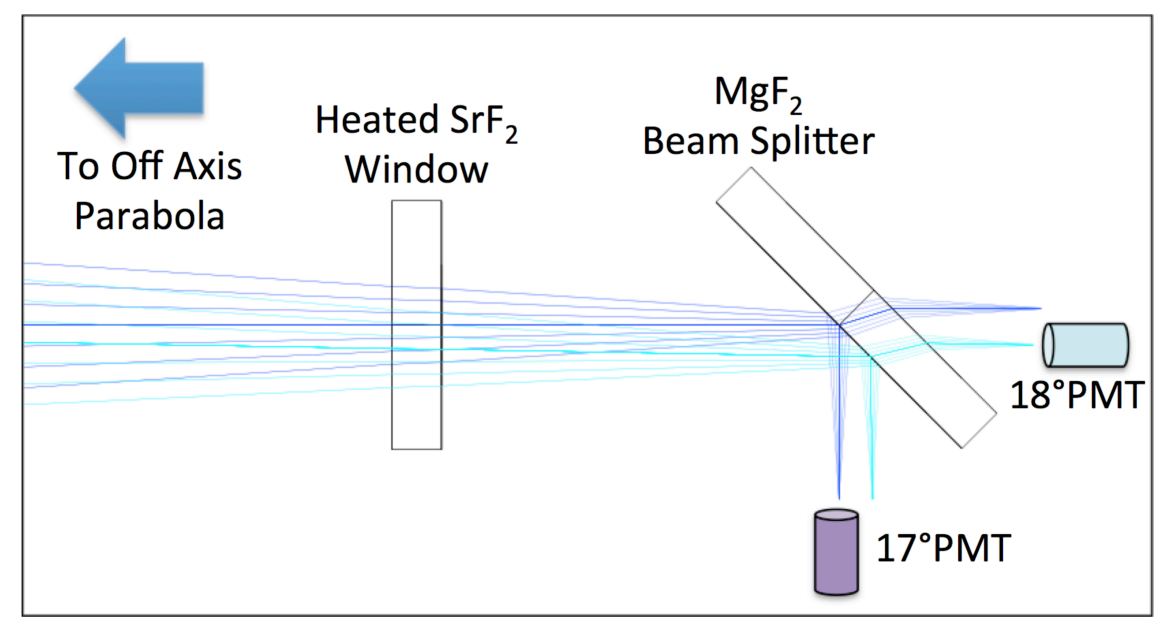

Figure 8. Ray trace of light bundles from $17^{\circ}$ down (dark blue, $\sim 200 \mathrm{~km}$ ) and $18^{\circ}$ down (light blue, $\sim 163 \mathrm{~km}$ ) depicting how the two PMTs can observe two different tangent point locations. 


\subsection{Expected Response from Tri-TIP}

An analysis was performed to determine the expected response from the Tri-TIP sensor for the Earth's nightglow spectrum $^{5}$. The study was performed using a nightglow and dayglow (for reference) spectra from the Ultraviolet Limb Imager acquired on May 5,1991 at 23:29:11 UT and May 4, 1991 at 7:33:05 UT respectively. These data are presented in Figure 9 panel 1, with the purple line representing the nightglow and the black line representing the dayglow scaled by a facto of 0.25 . The second panel represents what the PMT would see after a single reflection from the off axis parabola. The third panel takes into account the transmission effects of the heated strontium fluoride filter, both spectra have now been normalized at the $135.6 \mathrm{~nm}$ peak for comparative purposes. Panel 4 shows what is expected at the red leak tube after the light has passed through the sapphire beam splitter. Finally the fifth panel depicts the expected results after final post processing and subtraction of the red leak spectra (panel 4) from the UV spectra (panel 3).

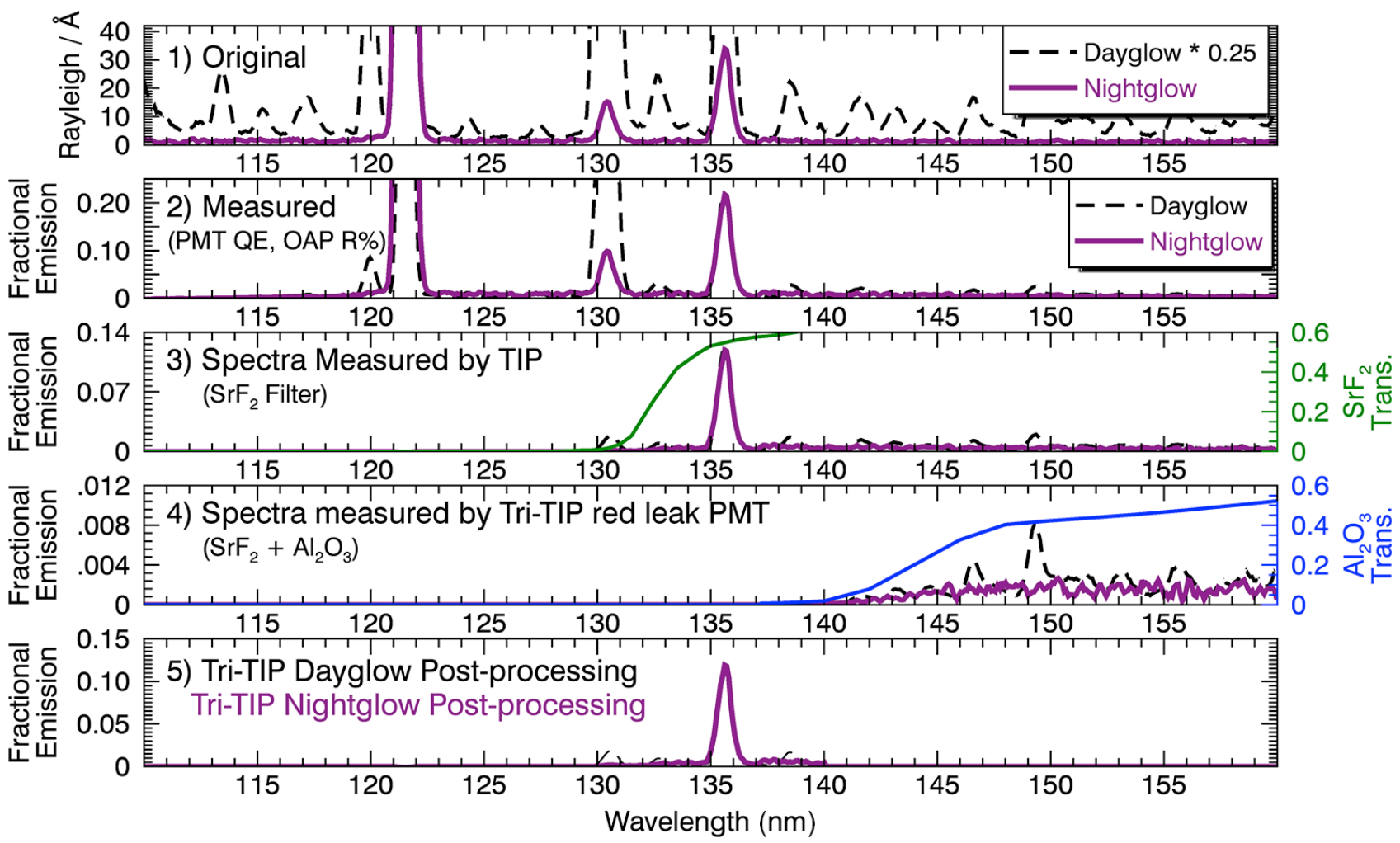

Figure 9. Optical effects of the Tri-TIP instrument through different phases of the optical path ${ }^{5}$, see text for details.

\section{IRIS SUITE}

The UK contribution to the Co-ordinated Ionospheric Reconstruction CubeSat Experiment (CIRCE) mission, is the Insitu and Remote Ionospheric Sensing (IRIS) suite. IRIS comprises three payloads, and is designed to make in-situ ionospheric particle \& radiation measurements, combined with remote sensing of GPS signals to map the ionosphere. Figure 10 shows a CAD model of the IRIS suite.

The three IRIS payloads showcase UK academia: (i) an ion and neutral mass spectrometer (INMS), from University College London's Mullard Space Science Laboratory (MSSL); (ii) a radiation monitoring package (RadMon), from Surrey Satellite Technology Limited (SSTL), with expertise drawn from the University of Surrey, and (iii) a GPS receiver (TOPCAT) that uses signal propagation delay to map the ionosphere from the University of Bath. 
It is anticipated that the IRIS data sets will:

(i) improve our understanding of the variability of atmospheric drag, the chemistry of the thermosphere and the impact of space weather on the upper-atmosphere;

(ii) identify areas of increased radiation that satellites should avoid, and help identify suitable orbits and shielding requirements for future satellites;

(iii) validate the Multi-Instrument Data Analysis System (MIDAS) tomography algorithm for the topside ionosphere and plasmasphere, by using total electron content (TEC) measurements from the differential phase of GPS signals, and inverting them to derive the electron density of the region.

In addition to being of interest in their own right, the individual IRIS sensors have been selected to provide contextual environmental information to the CIRCE mission. The data collected by IRIS will enrich the science that can be derived from the Tri-TIP UV photometry measurements, for tomographic specification of electron density.

Two identical IRIS flight units are provided for CIRCE. IRIS occupies the first $\sim 2 \mathrm{U} \times 1 \mathrm{U}$ of the lead CIRCE satellite bus, and the rear $\sim 2 \mathrm{U} \times 1 \mathrm{U}$ of the trailing bus.

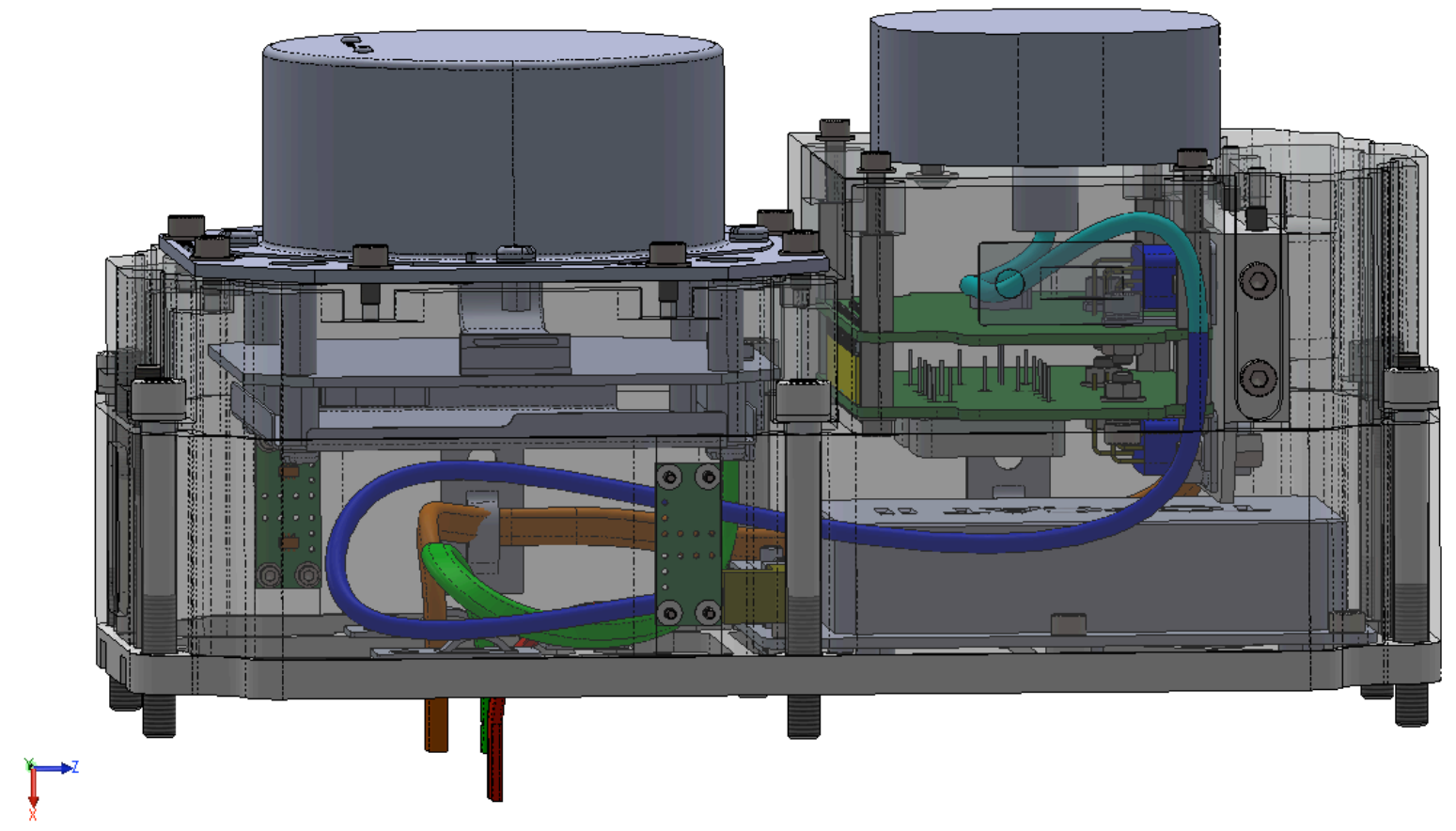

Figure 10: CAD model of the IRIS suite, comprising the ion and neutral mass spectrometer (top left), the TOPCAT GPS antenna (top right), the radiation monitoring package (center right), and the TOPCAT GPS receiver payload (bottom right).

\subsection{Ion and Neutral Mass Spectrometer (INMS)}

Designed and developed by University College London's MSSL, the Ion and Neutral Mass Spectrometer (INMS), is a miniaturised analyser capable of measuring densities of ionised and neutral particles in the ram direction of the host spacecraft. It is particularly optimised for resolving $\mathrm{O}^{+}, \mathrm{O}_{2}, \mathrm{NO}, \mathrm{N}_{2}$ concentrations, and ions, in the thermosphere ${ }^{6}$. 


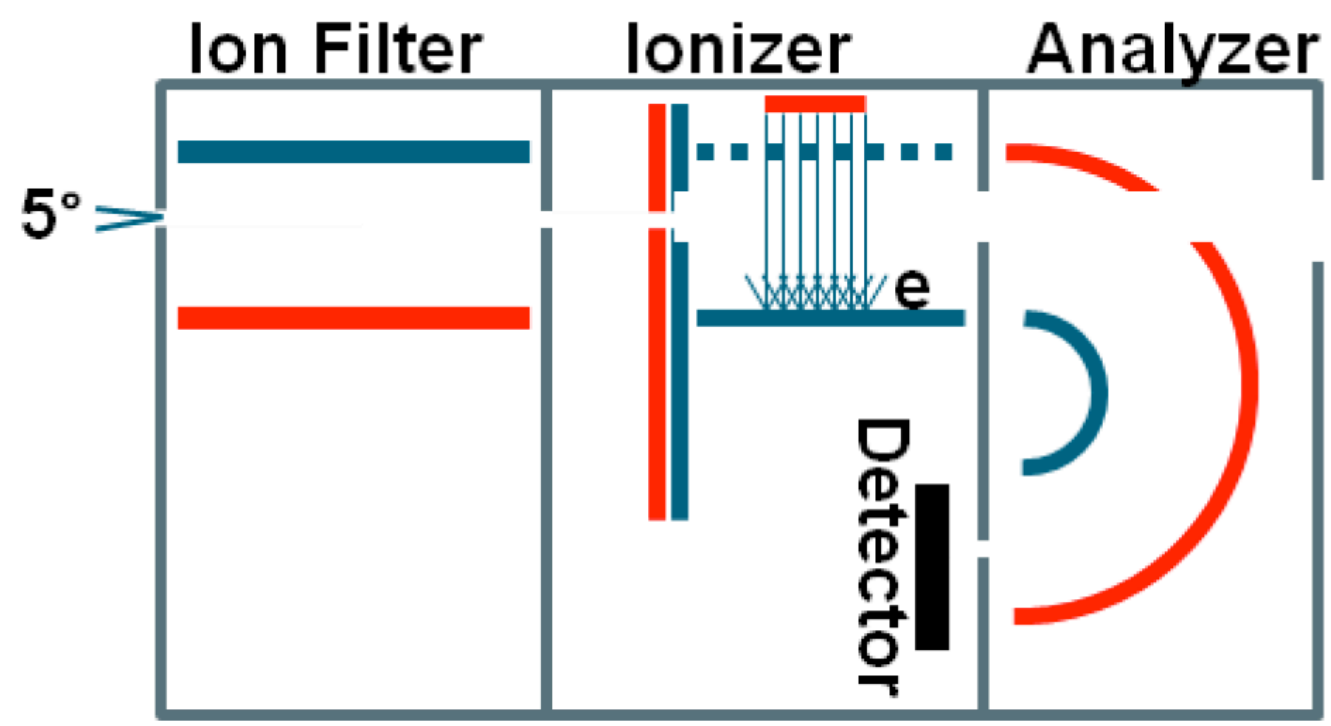

Figure 11: Overview schematic of INMS principle components and structure.

The instrument consists of a collimator, an ioniser and a charged particle spectrometer (see Figure 11). The ioniser consists of an electron source, an energy selector and a beam steerer, which can steer a beam of $50 \mathrm{eV}$ electrons into the charge exchange region. Voltages can be applied and removed in the collimator region to measure either neutral or charged particles. If voltage is applied, the charged particles entering the aperture will be rejected in the collimator. Neutral particles will become ionized as a result of this applied voltage, and will pass to the spectrometer for subsequent energy analysis. If no voltage is applied, neutral particles will simply pass through a gap in the spectrometer, which in turn will measure the energy of incoming charged particles.

Two INMS sensors will be utilised for the CIRCE mission (see Figure 12) one on each spacecraft. The INMS installed in the leading spacecraft will be permanently facing the ram direction. The trailing satellite will regularly rotate on its axis (twice an orbit) to enable the second INMS instrument to collect data at multiple spacecraft directions, when Tri-TIP is not operating.

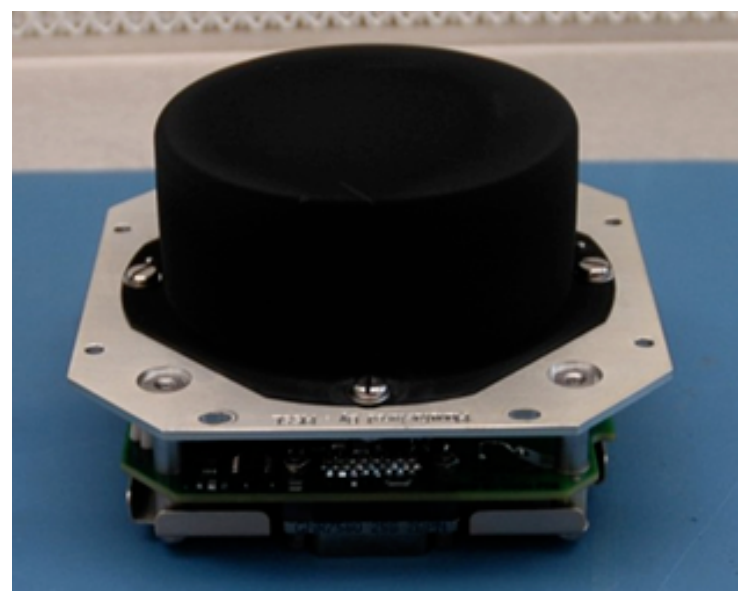

Figure 12: The ion and neutral mass spectrometer (INMS). 


\subsection{RadMon}

The Surrey Satellite Technology Ltd (SSTL) RadMon 3.0 is a development of the radiation monitor designs that originated from the Surrey Space Centre (SSC), a part of the University of Surrey.

Solar radiation and charged particle impacts, resulting from the solar wind and solar storms can have a damaging effect on spacecraft materials and electronics ${ }^{7,8}$. These effects can be instantaneous as energetic heavy ions and protons pass through the spacecraft structure and instrumentation causing single-event effects (SEEs). Additionally, long duration exposure to the space environment can cause long term degradation of materials as the radiation dose accumulates over time ${ }^{9}$.

As commercial-off-the-shelf (COTS) components become more common place, particularly within the small satellite industry, it has become increasingly important to understand the effect the space environment has on new components that are not typically designed for use in space, and have little, if any, space flight heritage $e^{9,10}$.

To date, six spacecraft have flown the SSTL/SSC developed radiation monitors in LEO, MEO and GEO orbits. In addition to providing the technology with spaceflight heritage, the monitors have also provided a near continuous data set of the geomagnetic radiation environment since $1992^{11}$.

The latest generation RadMon 3.0 for CIRCE/IRIS is a near real time, low mass, volume and power instrument that can provide the operator with information on the radiation environment local to the spacecraft. RadMon 3.0, shown in Figure 13, is capable of highlighting any radiation impact that exceeds a predefined threshold, a value that can be configured both prior to launch and while on orbit. This capability allows operators to react appropriately to the radiation environment encountered by the spacecraft, initiating mitigation strategies to protect the asset against the environment ${ }^{11}$.

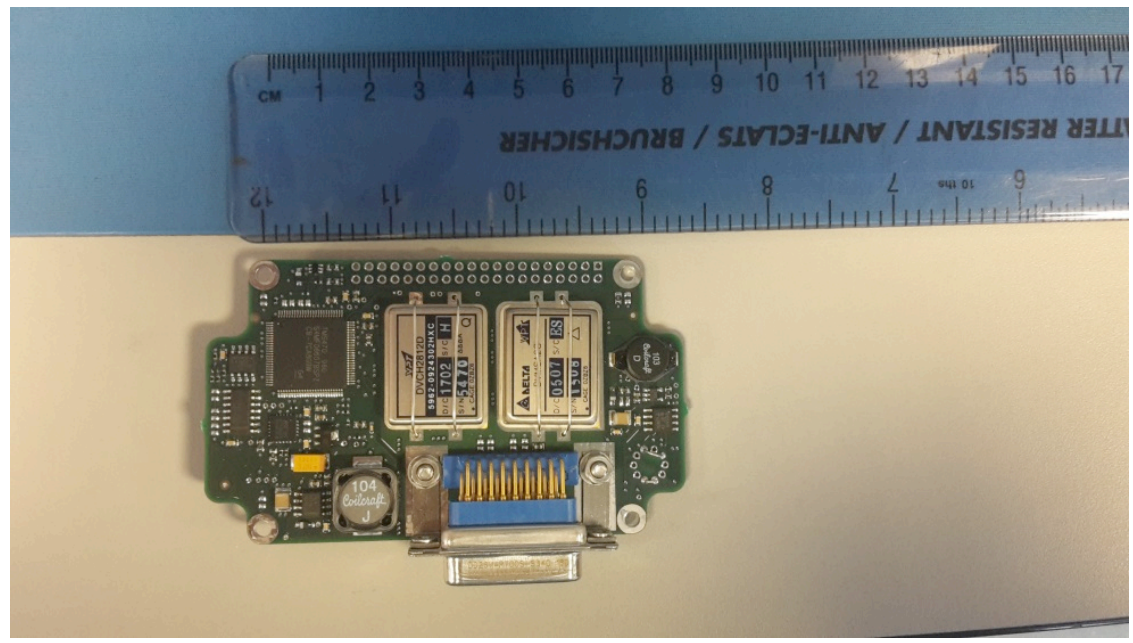

Figure 13. RadMon 3.0 engineering model, power and communications board.

The CIRCE RadMon 3.0 instrument will incorporate three RadFET sensors to measure total ionising radiation dose: one internal sensor, and two externally mounted within the IRIS volume. An Ultraviolet (UV) photodiode is used for dose rate monitoring, and a large area PIN diode detector is used to measure proton and heavy ion fluxes. Each of the CIRCE spacecraft will host an identical RadMon 3.0 configuration. Two instruments in the same orbit will provide useful crosscalibration of the sensors as well as day to day inner radiation belt dynamics at $500 \mathrm{~km}$. Data collection and correlation with spacecraft system upsets and anomalies will help identify the on-board systems most at risk, and provide a guide to the resilience of the COTS components on the spacecraft.

The data from the RadMon 3.0 instruments will be beneficial to radiation models, improving the accuracy of boundary values and predictions. In addition to monitoring the local radiation environment it is anticipated that RadMon 3.0 will demonstrate particle flux and energy increases in line with Space Weather events, adding to the instrument's capabilities in monitoring space weather impacts. 


\subsection{TOPCAT}

The TOPside ionosphere Computer Assisted Tomography (TOPCAT), designed at the University of Bath, uses the delay in dual-frequency GPS signals to map the ionosphere.

Measuring the total electron content (TEC) between GPS satellites and CIRCE will provide a new dataset that can directly improve the vertical resolution of current ionospheric and plasmaspheric imaging.

TOPCAT I was part of a PhD project and technology demonstrator designed at the University of Bath from commercialoff-the-shelf (COTS) components, to deliver a relatively cost-effective means of conducting upper atmospheric science. TOPCAT I was installed on-board the CubeSat "UKube-1", launched 2014 ${ }^{12}$. The TOPCAT II design for CIRCE/IRIS comprises a Novatel GPS receiver, a payload controller board, and utilizes a dual-frequency AntCom G5 Antenna.

The receiver used in TOPCAT II is the commercially available Novatel OEM 719 module, customized for space applications. It has an increased Doppler bandwidth, enabling satellite tracking at high velocities of $\sim 7 \mathrm{~km} / \mathrm{s}$. The elevation cut-off functionality is disabled, as a reference to elevation is not available when tracking satellites from spacebased receivers. A TOPCAT engineering model is shown in Figure 14. The receiver is connected to an active L1/L2 dual-frequency Antcom G5 antenna manufactured for aerospace applications, which also picks up the L5 frequency.

To deliver a robust payload that allows maximum possible scientific output under varying operational conditions, flexibility is included in TOPCAT's functionality. Multiple operational modes have been implemented with different features, together with error and parameter handling that enable status monitoring of the payload and the ability to respond when required. These features offer some control over the instrument in an otherwise fully automated system.

As a payload on-board CIRCE, TOPCAT II provides a unique geometry of measurements to view our near-Earth space environment. Imaging the ionosphere using tomography has been a well-established concept since it was first demonstrated with LEO satellites of the U.S. Navy Ionosphere Monitoring System (NIMS), and the Russian CICADIA satellites ${ }^{13}$. Ionospheric (TEC) tomography uses the integral of the number of free electrons between satellite and receiver, measured by radio signals propagating through the medium. For GPS-based tomography, TEC measurements are derived from the differential phase of dual-frequency transmissions of GPS satellites. The final TEC allows fourdimensional (4-D - i.e. 3-D spatial and time evolving) mapping of the ionosphere ${ }^{14}$.

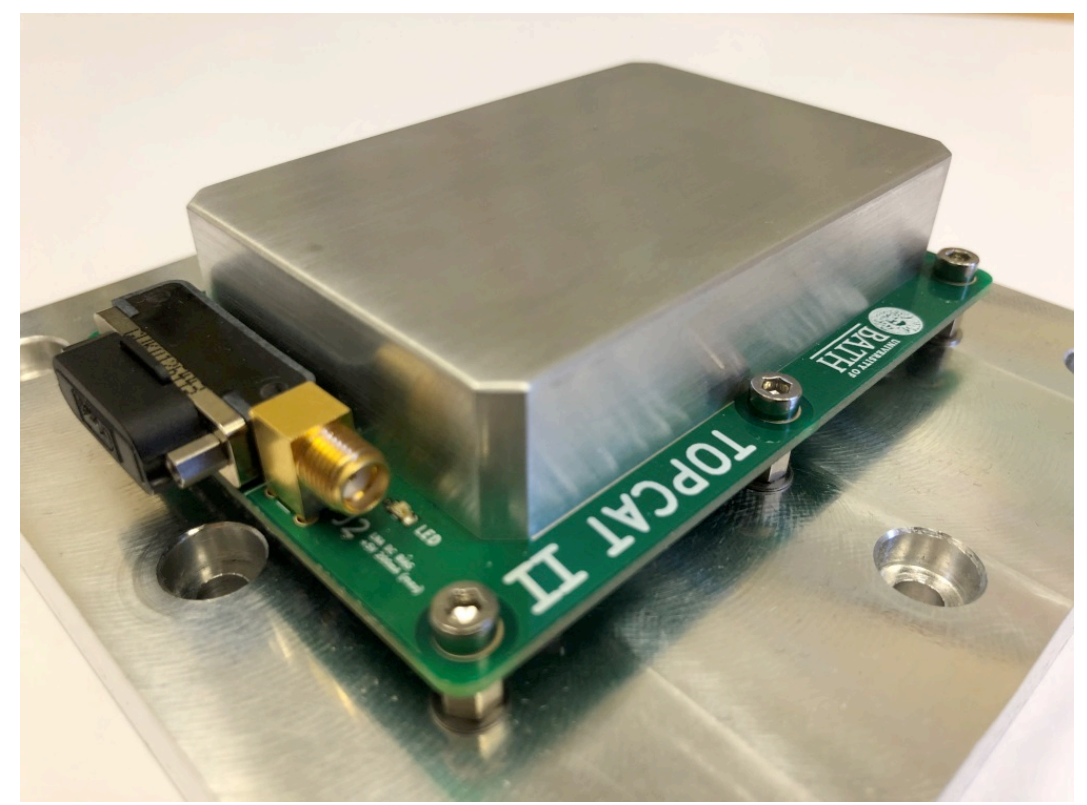

Figure 14. Photograph of the TOPCAT II engineering model. 


\section{SUMMARY}

The CIRCE mission is pushing the boundaries of the CubeSat platform technology as well as the ability to collect highsensitivity ionospheric remote-sensing data from such platforms. This multi-national collaboration has resulted in five space weather payloads on each of the $6 \mathrm{U}$ CubeSats, challenging the size, weight and power constraints of the platform as well as integration and complex concepts of operations. There are multiple scientific synergies between the individual payloads, as well as compelling science to be obtained from the short spatial and temporal cadence of measurements between the two spacecraft.

\section{ACKNOWLEDGEMENTS}

The CIRCE mission is a jointly-funded program between the US Naval Research Labs and Dstl. Work at NRL for all authors is supported by the chief of Naval Research. The UK's IRIS payloads acknowledge funding from Dstl's Space programme. Content includes material subject to (C) Crown copyright (2019), Dstl. This material is licensed under the terms of the Open Government Licence except where otherwise stated. To view this licence, visit http://www.nationalarchives.gov.uk/doc/open-government-licence/version/3 or write to the Information Policy Team, The National Archives, Kew, London TW9 4DU, or email: psi@nationalarchives.gov.uk

\section{REFERENCES}

[1] Meier, R. R., "Ultraviolet spectroscopy and remote sensing of the upper atmosphere," Space Sci. Rev. 58, 1-185 (1991).

[2] Dymond, K. F. et al., "The Tiny Ionospheric Photometer (TIP) on the constellation observing system for meterology, ionosphere, and climate (COSMIC/GORMOSAT-3)," J. Geophys. Res. A Space Phys. 121, 10,614$10,622(2016)$.

[3] Budzien, S. A., et al., "Tiny Ionospheric Photometers on FORMOSAT-3/COSMIC: on-orbit performance," Proc. SPIE 7438, 743813 (2009).

[4] Stephan, A. W., et al., "Evaluation of UV optics for Triple Tiny Ionospheric Photometers on CubeSat missions," Proc. SPIE 10769, 107690W (2018).

[5] Fritz, B. A. , et al., "Ultraviolet beam splitter characterization for use in a CubeSat optical system," J. Appl. Remote Sens. 13(3),032503 (2019).

[6] Kataria, D. et al., "QB50 INMS User Manual”, QB50-INMS-MSSL-UM-13002_INMS_User_Manual_Issue_3, 2015.

[7] Fleetwood, D.M., et al., "An overview of radiation effects on electronics in the space telecommunications environment", Microelectronics Reliability, 40 (2000) 17-26, (2000).

[8] Cannon, P., et al., "Extreme space weather: impacts on engineered systems and infrastructure", Royal Academy of Engineering, ISBN 1-903496-95-0, February (2013).

[9] Underwood, C., et. al., "Development of Miniaturised Radiation Environment Monitors for Commercial Spaceflight: Flight Results from the MuREM/RM Payloads", IEEE, 978-1-5090-4366-8/16, (2016).

[10] Kayali, S., "Utilization of COTS Electronics in Space Application, Reliability Challenges and Reality", Jet Propulsion Laboratory, California Institute of Technology, (2002).

[11] SSTL RadMon Radiation Monitor Datasheet.

[12] Pinto-Jayawardena, T., "Topside Ionosphere/Plasmasphere Tomography Using Space-Borne Dual Frequency GPS Receivers", Ph.D., University of Bath, (2015).

[13] Leitinger, R., et al., "Electron content measurements with geodetic Doppler receivers", Radio Science, Vol 19, Issue 3,685 , (1984).

[14] Bust, G. S., and C. N. Mitchell, "History, current state, and future directions of ionospheric imaging", Rev. Geophys., 46(1), (2008). 